\title{
Concentración de inmunoglobulina $G$ en calostro bovino en cruces Bos taurus x Bos indicus en los primeros tres días pos parto
}

\author{
Immunoglobulin G concentration in bovine colostrum of Bos taurus $x$ \\ Bos indicus crosses during the first three days postpartum
}

\section{Concentração de imunoglobulina $\mathrm{G}$ no colostro bovino em cruza Bos taurus $x$ Bos indicus nos três primeiros dias após o nascimento.}

\author{
Leidy J. Reyes-Castañeda ${ }^{1 *}$, Jorge L. Parra-Arango ${ }^{2 *}$, Hernando Flórez-Díaz $^{3 *}$ \\ 1* MVZ, MSc; \\ 2 DMV, MSc Facultad de Ciencias Agropecuarias y Recursos Naturales, Universidad de los Llanos, Vereda \\ Barcelona. Kilómetro 12 vía Puerto López, \\ 3* MVZ, MSc, PhD, Corpoica La Libertad. Kilómetro 17 vía Puerto López. \\ * Grupo de investigación GIRGA, Universidad de los Llanos \\ Email: jlparra@unillanos.edu.co
}

Recibido: septiembre 21 de $2015 \quad$ Aceptado: mayo 30 de 2016

\begin{abstract}
Resumen
El objetivo fue correlacionar la concentración de inmunoglobulina G (IgG) con la proteína, los minerales y el color en 69 calostros de bovinos de cruces Bos taurus x Bos indicus de un predio del sistema de producción doble propósito del piedemonte llanero en los primeros tres días posparto. La concentración de IgG en calostro presentó una correlación negativa con los días posparto, y una correlación positiva con el contenido de proteína, lactosa y sólidos en calostro entero. La correlación entre el color del calostro entero y la concentración de lgG fue positiva para el valor b* $(r=0.182, p=0.024)$, localizado entre el rango de azules y amarillos. La concentración mediana de lgG para el día uno fue 51,601 mg/ml, para el día dos de $23,319 \mathrm{mg} / \mathrm{ml}$ y para el día tres de $36,534 \mathrm{mg} / \mathrm{ml}$. Las concentraciones de IgG fueron significativamente superiores el día 1 posparto e inversamente correlacionada con los días posparto $(p<0,05)$, el número del parto no se encontró asociados a la concentración de $\operatorname{lgG}(p>0,05)$. La población bovina evaluada produce calostro de buena calidad que puede aprovecharse para la transferencia de inmunidad pasiva creando bancos de calostro.
\end{abstract}

Palabras clave: IgG, ELISA, parto, color. (AIMS, FAO)

\begin{abstract}
This study was aimed to correlate the immunoglobulin G (IgG) concentration with protein, minerals and color in 69 bovine colostrums from Bos taurus x Bos indicus crosses from a dual-purpose production system farm on Colombia in the piedemonte llanero during the first three days postpartum. IgG concentration in colostrum had a negative correlation with days postpartum whereas a positive correlation was found with protein, lactose and solid content in whole colostrum. The correlation between whole colostrum color and IgG concentration was positive for the $b^{*}$ value $(r=0.182, p=0.024)$, located it in the range of blues and yellows. Mean IgG concentration for day one was 51,601 mg/ml, 23,319 mg/ml for
\end{abstract}


day two and $36,534 \mathrm{mg} / \mathrm{ml}$ for day three. IgG concentrations were significantly higher on day 1 postpartum and inversely correlated with days postpartum $(\mathrm{p}<0.05)$. The number of calvings was not associated with IgG concentration ( $p>0.05)$. The bovine population evaluated produced good quality colostrum which can be used for transfer of passive immunity by using colostrum banks.

Keywords: Immunoglobulin, ELISA, parturition, colour (AIMS, FAO)

\section{Resumo}

O objetivo foi correlacionar a concentração de imunoglobulina G (IgG) com proteína, minerais e cor em 69 amostras de colostro de bovinos da cruza Bos taurus x Bos indicus de um sistema de produção de dupla aptidão do Piedemonte llanero nos primeiros três dias pós-parto. A concentração de lgG no colostro apresentou correlação negativa com os dias pós-parto, e uma correlação positiva com o teor de proteína, lactose e sólidos no colostro total. A correlação entre a cor de colostro todo e a concentração de IgG foi positivo para o valor $b^{*}(r=0,182, p=0,024)$, localizado entre a gama de azuis e amarelos. A concentração mediana de IgG dia um foi 51,601 mg / ml, para o dia dois de 23,319 mg / ml e para o dia três de 36,534 mg / ml. As concentrações de IgG foram significativamente maiores no dia um pós-parto e inversamente correlacionado com os dias pós-parto $(p<0,05)$, para o número de parição não foi encontrada associada com a concentração de $\lg G(p>0,05)$. A população de gado avaliada produz colostro de boa qualidade que pode ser utilizado para a transferência de imunidade passiva a partir de bancos de colostro.

Palavras chave: IgG, ELISA, parto, cor. (AIMS, FAO).

\section{Introducción}

El manejo del ternero recién nacido incluye: supervisión y asistencia al parto; protocolo de reanimación cuando es necesario, cierre de cordón umbilical, monitoreo sanitario perinatal, ambiente higiénico, seco y ventilado (Mee, 2008).

El protocolo perinatal involucra el acceso oportuno a calostro de buena calidad, para suministrar nutrición e inmunidad frente a potenciales infecciones del período perinatal y neonatal (Chase et al., 2008b).

Las propiedades inmunológicas del calostro se deben a las concentraciones de inmunoglobulinas solubles (Elfstrand et al., 2002, Pandey, 2011), principalmente ta IgG (Liu et al., 2009, Baumrucker et al., 2010), que están relacionadas con el número y la etapa de lactancia, la producción láctea, el recuento de células somáticas y el contenido de proteína en la leche.

La IgG se encuentra en linfa, sangre, suero sanguíneo, leche, lactosuero y calostro bovino. La IgG1 es la principal inmunoglobulina en las secreciones salival y láctea y en la transferencia al neonato (Butler, 1969)

La viabilidad del neonato depende de una exitosa transferencia de inmunidad pasiva de origen materno a través del calostro en las primeras 6 horas de vida. La eficiencia de la transferencia se puede evaluar con dos pruebas de campo a saber: por refractómetro para la evaluación de proteína y la prueba de turbidez de sulfito de sodio con suero del recién nacido, o pruebas de laboratorio como colorimetría, electroforesis e inmunodifusion radial, o enzimas indicadoras como Gama Glutamil Transferasa (GGT) y fosfatasa alcalina (Weaver et al., 2000).

El calostro presenta importantes solutos, minerales, vitaminas, inmunoglobulinas (Christiansen et al., 2010), factores de crecimiento, citoquinas, leucocitos (Elfstrand et al., 2002, Hagiwara et al., 2000). Todos estos componentes están correlacionados con la calidad inmunológica, partiendo del punto de corte de un calostro de buena calidad con concentraciones superiores a $50 \mathrm{mg} \mathrm{lgG/ml} \mathrm{de} \mathrm{calostro} \mathrm{(Flórez} \mathrm{et} \mathrm{al.,} \mathrm{2000).} \mathrm{El}$ propósito del presente estudio fue evaluar la concentración de inmunoglobulina $\mathrm{G}$ en calostro bovino en los primeros tres días posparto en cruces de vacas Bos taurus $x$ Bos indicus y asociarlo a la concentración de minerales y la clasificación colorimétrica del mismo.

\section{Materiales y métodos}

La investigación se realizó en un predio del municipio de Castilla La Nueva, Departamento del Meta (N3-48.664; W73 43.378), que en promedio ordeña 150 vacas por día. Fue seleccionado por conveniencia en razón del número de vacas en ordeño y el ingreso constante de vacas en periodo de calostro. El ordeño era mecánico con apoyo del ternero. El manejo reproductivo era asistido por inseminación artificial. La diversidad racial consiste en cruces en proporciones indeterminadas de Bos indicus de la raza Gyr por Bos taurus de las razas, Holstein negro y rojo, Pardo Suizo y en menor proporción Blanco Orejinegro. La alimen- 
tación era en pastoreo en las gramíneas Brachiaria decumbens, Brachiaria humidícola, gramas, leguminosas nativas y suplementación con una mezcla de sal mineralizada, alimento comercial y palmiste a razón de 0,5 $\mathrm{Kg} / \mathrm{vaca} /$ día en ordeño.

\section{Tamaño de muestra y muestreo}

El tamaño de la muestra se determinó con Epidat 4.1, asumiendo una desviación estándar para IgG en calostro de $5,5 \mathrm{mg} / \mathrm{ml}$, un nivel de confianza del $95 \%$ y una precisión de 1,3 mg/ml. Obteniéndose un total de 69 calostros, el muestreo fue sistemático, distanciados entre sí por 4 días, tomando las muestras de calostro del 2 y 3 día posparto antes del amamantamiento de los terneros y del día 1 post amamantamiento. Se colectaron 69 calostros con volúmenes de $300 \mathrm{ml}$ por vaca, en frascos de vidrio estériles, que se transportaron en cavas isotérmicas hasta el laboratorio de salud animal de Corpoica en el CI La Libertad. Se tomaron 10 muestras de leche comercializable de la misma finca como control, previa homogenización de las cantinas, al final del ordeño de las 150 vacas en producción.

\section{Determinación de IgG}

Se dispusieron $50 \mathrm{ml}$ de calostro homogenizado en tubos Falcon ${ }^{\circledR}$, se centrifugó a 800 gravedades (g) por 20 minutos a $8{ }^{\circ} \mathrm{C}$ y se dejó en refrigeración a $5^{\circ} \mathrm{C}$ por 24 horas, procediendo a extraer la grasa con una espátula. El volumen restante se incubó en baño de María hasta alcanzar $37^{\circ} \mathrm{C}$ de temperatura, seguidamente se adicionó $1 \mathrm{ml}$ de renina industrial, se dejó coagular a temperatura ambiente $\left(22^{\circ} \mathrm{C}\right)$ por dos horas, se hizo cuadrícula del coagulado con espátula, se centrifugó a 800 g por 20 minutos a $8{ }^{\circ} \mathrm{C}$, se midió el volumen del suero de calostro y se almacenaron dos alícuotas de $2 \mathrm{ml}$ en crioviales estériles. El suero se conservó a $-20^{\circ} \mathrm{C}$ hasta la determinación de IgG.

Brevemente con el Bovine Reference Serum, RS10103-5 (kit Bovine IgG, Bethyl®, USA) se obtuvieron por diluciones dobles, 8 concentraciones descendentes de IgG desde $500 \mathrm{mg} / \mathrm{ml}$ hasta $7,8 \mathrm{mg} / \mathrm{ml}$; la concentración de referencia sin IgG, se hizo con agua destilada, desmineralizada y desionizada. Se sensibilizaron cajas de poliestireno de 96 celdas, dos columnas de celdas para diluciones patrón por duplicado para obtener la curva estándar y las restantes columnas se sensibilizaron para las muestras de suero de calostro, el proceso se completó por 1 hora a $22^{\circ} \mathrm{C}$. Como conjugado se utilizó $100 \mu \mathrm{l}$ de HRP Detection antibody, con una dilución de 1:100,000 del HRP Conjugated Bovine Ig Detection Antibody. Se siguieron las instrucciones del fabricante, la lectura de absorbancia se hizo a $450 \mathrm{~nm}$ en el lector de Elisa Biotek (EON, USA).

Con las 8 concentraciones de IgG del Kit, se construyó una curva patrón, de la concentración en función de la absorbancia, para intrapolar las lecturas de las muestras de suero de calostro.

Teniendo en cuenta el volumen promedio de suero de calostro extraído, de los $50 \mathrm{ml}$ de calostro iniciales, se obtuvo un factor de multiplicación de 6,7 para el día 1; 5,5 para el día 2 y 5 para el día 7, con el cual se restituyeron las concentraciones de $\lg G$, de la prueba de Elisa en suero, al volumen inicial de la muestra de calostro.

\section{Otras pruebas analíticas}

Para realizar el análisis de proteína se diluyó 1:4 al tomar $5 \mathrm{ml}$ de calostro en un vaso de precipitado y adicionando $15 \mathrm{ml}$ de agua destilada, desmineralizada y desionizada; obteniendo el resultado en porcentaje (p/p) g/100 g de calostro mediante ultrasonido en el equipo Ekomilk® (Bulgaria).

Para el análisis de macro y microminerales una submuestra de $50 \mathrm{ml}$ de calostro depositada en un tubo Nalgeno (BD Falcon, USA) se congeló $-8^{\circ} \mathrm{C}$ hasta el envió al laboratorio de suelos de Corpoica en el Centro de investigaciones sede Tibaitatá (C.I Tibaitatá) para la determinación de minerales.

Los macrominerales en porcentaje $(p / p)$ g/100 g de calostro fueron: calcio, fosforo, magnesio, azufre, potasio, sodio. A su vez los microminerales en partes por millón (ppm) fueron: hierro, cobre, manganeso y zinc.

Los elementos calcio, magnesio, potasio, sodio, hierro, cobre, manganeso y zinc se determinaron por absorción atómica. El fosforo total por el método Bary II y determinación colorimétrica por espectrofotometría mientras que el azufre se analizó por turbidez con cloruro de Bario y cuantificación por espectrofotometría.

El análisis de color se realizó en una caja de Petri depositando $50 \mathrm{ml}$ de calostro entero tomando por triplicado los datos para cada muestra con espectrofotómetro (Hunter MiniScan XE, Model 45/0-L, Hunter Associates Laboratory Inc., Reston, VA, USA).

El espectrofotómetro entrega tres medidas a saber: $L^{*}$, $a^{*} y b^{*}$, representadas en un eje tridimensional, donde $L$ en el eje longitudinal vertical ( $L$ de 0 a 100) representa la luminosidad de la muestra, $a^{*}$ representa la posición relativa del color entre verdes (a-) y rojos $(\mathrm{a}+)$, 
y b* representa la posición relativa entre azules (b-) y amarrillos $(b+)$.

Los resultados sobre calidad, proteína, minerales y color por espectrometría se presentan en otra publicación, en el presente trabajo estas variables se utilizaron para correlacionarlas con las concentraciones de IgG

\section{Análisis estadístico}

Se efectuó análisis de distribución de los datos, para la hipótesis de normalidad de la concentración de IgG mediante la prueba de Shapiro-Wilk, si la variable presenta distribución normal, el análisis incluye estadística descriptiva y procedimientos paramétricos como análisis de varianza de una vía y correlación bivariada de Pearson. Ante la no normalidad de la variable, se efectúan pruebas no paramétricas análogas.

Se calcularon estadísticos de tendencia central con media y mediana y de dispersión con desviación estándar y coeficiente de variación. La comparación de medianas de IgG, por número del parto y día posparto, se hizo con la prueba no paramétrica de Kruskall-wallis para $\mathrm{K}$ muestras independientes, cuando el resultado fue significante $(p<0,05)$ se efectuó la prueba de Mann Whitney para muestras independientes entre pares de factores, teniendo en cuenta que la significancia 0,05 se dividía por el número de niveles del factor $(0,05 / 3=$ $0,017)$, para los días de calostro con el fin de no incrementar el error tipo I. Las correlaciones entre IgG en calostro con, grasa, proteína, color, minerales se hicieron con correlación bivariada de Spearman. El análisis estadístico se corrió con el software SPSS-18 (2014).

\section{Resultados y discusión}

La concentración de IgG en calostro, no presentó distribución normal, presentando desviaciones estándar superiores o cercanas a la media que se traducen en altos coeficientes de variación, por lo cual los procedimientos estadísticos utilizados correspondieron a estadística no paramétrica. Tabla 1

La concentración mediana de $\lg$ G en calostro para los primeros 3 días posparto fue: 51,$601 ; 23,319$ y 36,534 $\mathrm{mg} / \mathrm{ml}$ respectivamente Tabla 1 . Las concentraciones de IgG son superiores a los de Oyeniyi y Hunter (1978), Foley y Otterby (1978), Vann et al. (1995) y Gulliksen et al., (2008) quienes reportaron concentraciones de $41,32,50$ y $45 \mathrm{mg} \mathrm{lgG} / \mathrm{ml}$ de calostro, respectivamente. $Y$ son inferiores a Muller y Ellinger (1981), Tyler et al. (1999a) y Kehoe et al., (2011), reportando 92, 97 y $100 \mathrm{mg} \mathrm{IgG/ml} \mathrm{de} \mathrm{calostro,} \mathrm{respectivamente.} \mathrm{Kehoe}$ et al (2011) evaluaron a las 2 y 6 horas posparto incrementando las expectativas de encontrar mayores concentraciones de IgG, para ellos el $10 \%$ del total de las muestras evaluadas presentaban concentración superior al punto de corte del calostro de buena calidad y $43 \%$ era mayor o igual a $100 \mathrm{mg} / \mathrm{ml}$.

La concentración mediana de $\lg G$ para el día 2 correspondió al $50 \%$ de la del día 1, Tabla 1. En el piedemonte llanero y para el mismo sistema de producción, Hernández y Olaya (2004) encontraron una concentración de $\lg \mathrm{G}$ en calostro de $58.82 \mathrm{mg} / \mathrm{ml}$.

El día 3 presentó una concentración mediana mayor a la del día dos sin observar diferencias significativas entre días posparto. Para este día se observó una distribución más homogénea de la concentración de lgG en los animales muestreados indicando que para este día existe una reducción en la variabilidad de la concentración de calostro.

Se considera un calostro de buena calidad aquel que presenta concentraciones $\geq 50 \mathrm{mg} / \mathrm{ml}$ (Flórez et al., 2000 ), en el presente estudio el $50 \%, 20 \%$ y $10 \%$ de los calostros de los 3 primeros días posparto respectivamente presentaron concentraciones $\geq 50 \mathrm{mg} / \mathrm{ml}$. Uno de cada cinco calostros del primer día posparto

Tabla 1. Estadígrafos de tendencia central y dispersión para concentraciones de lgG en calostro.

\begin{tabular}{|l|c|c|c|}
\hline \multirow{2}{*}{ Estadísticos Descriptivos } & \multicolumn{3}{|c|}{ Días de calostro } \\
\cline { 2 - 4 } & $\mathbf{1}$ & $\mathbf{2}$ & $\mathbf{3}$ \\
\hline Media $(\mathrm{mg} / \mathrm{ml})$ & 83,458 & 49,685 & 34,117 \\
\hline Mediana $(\mathrm{mg} / \mathrm{ml})$ & $\mathbf{5 1 , 6 0 1 a}$ & $23,319 \mathrm{a}$ & $36,534 \mathrm{a}$ \\
\hline Desviación estándar & 82,630 & 60,480 & 14,271 \\
\hline Coeficiente de variación $(\%)$ & 99 & 121 & 41 \\
\hline Shapiro wilk $(\mathrm{p})$ & 0,000 & 0,000 & 0,426 \\
\hline
\end{tabular}

Letras distintas señalan medianas diferentes 
(20\%) alcanzó concentraciones de IgG superiores a $155,7 \mathrm{mg} / \mathrm{ml}$.

El $30 \%$ de los calostros presentó concentraciones de IgG superiores a 95,4 mg/ml. La figura 1 presenta los percentiles de las concentraciones de IgG en los 69 calostros, independiente del día posparto de colecta.

La concentración mediana de IgG en calostro, presentó diferencias significativas entre días posparto, el número de partos no presentó diferencias estadísticas en las medianas (Tabla 2)

Difiriendo de Elfstrand et al., (2002) quienes reportaron entre los factores que afectan las concentraciones de IgG la edad de la vaca, la raza y el número de lactancias (Muller and Ellinger, 1981) e incluso el volumen del calostro producido.

La calidad del calostro medido en la concentración de IgG como lo reportaron Shoshani et al., (2014) no se ve afectada por un periodo seco corto. Por otra parte la frecuencia de los ordeños afecta la concentración de IgG, IgM e IgA como lo demostraron Hernández-

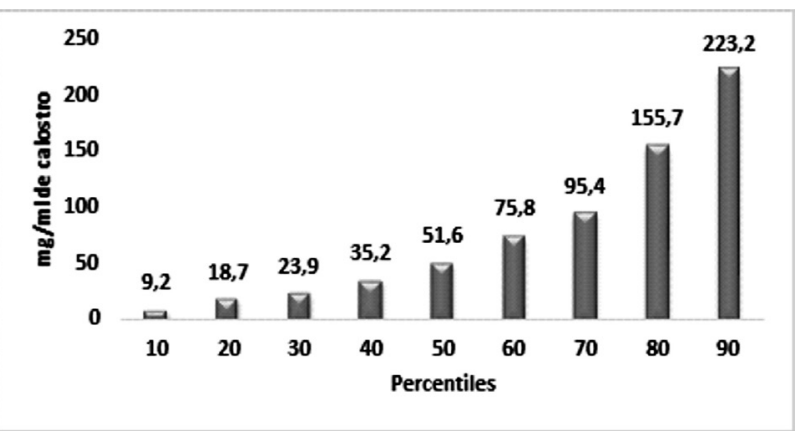

Figura 1. Percentiles de la concentración de $\lg G$ en 69 calostros.
Castellano et al., (2011) en calostro de cabra. La población bovina evaluada tuvo un periodo seco entre 70 a 90 días, y el calostro se obtuvo previa alimentación adecuada del neonato.

Difiriendo de lo encontrado por Kehoe et al., (2011), quienes encontraron que la media de concentración de IgG en calostro estaba influenciada por número de parto, superando el punto de corte de clasificación de un calostro de buena calidad de $50 \mathrm{mg} / \mathrm{ml}$. Al igual que Gulliksen et al., (2008) reportaron concentraciones mayores en vacas de más de cuatro partos.

La prueba de Mann Whitney mostró diferencias significativas $(p<0.05)$ para la concentración de IgG entre el día 1 y día 2 posparto. (Tabla 3)

La correlación de Spearman entre concentración de IgG y días posparto fue negativa y significante para los días posparto $(r=-0.241 ; p=0.046)$ correspondiendo con una decreciente concentración, a medida que los días posparto se incrementan. Así mismo la concentración de IgG en calostro presentó correlación positiva y significante con la concentración de proteína $(r=0.268 ; p=0.026)$ debido parcialmente a la naturaleza proteica de las IgG.

Resalta, la correlación significativa y directa de la IgG con la densidad del calostro $(r=0,239 ; p=0.048)$, con un coeficiente de correlación $(r)$ similar al presentado con las proteínas, indicando la utilidad de la densidad, para aplicaciones tecnológicas como el calostrometro, que se basa en estimaciones de la calidad inmunológica del calostro a partir de la densidad.

Las concentraciones de minerales en calostro presentaron correlaciones directas altamente significativas con las concentraciones de fósforo $(r=0,413, p=0.001)$, sodio ( $r=0,337, p=0.008)$, cobre $(r=0.268, p=0.036)$, así

Tabla 2. Prueba de Kruskall-wallis para concentración de IgG en calostro por día posparto y numero del parto.

\begin{tabular}{|c|c|c|c|c|c|c|}
\hline Factor & Niveles factor & $\mathbf{N}$ & $\begin{array}{c}\text { Rango } \\
\text { promedio }\end{array}$ & $\chi^{2}$ & gl & p-valor \\
\hline \multirow{3}{*}{ Días posparto } & \multirow{3}{*}{$\begin{array}{c}\text { Día } 1 \\
\text { Día } 2 \\
\text { Día } 3\end{array}$} & 32 & 41.06 & \multirow{3}{*}{5.825} & \multirow{3}{*}{2} & \multirow{3}{*}{0.05} \\
\hline & & 26 & 28.44 & & & \\
\hline & & 11 & 32.86 & & & \\
\hline \multirow{4}{*}{$\begin{array}{l}\text { Número } \\
\text { del parto }\end{array}$} & \multirow{4}{*}{$\begin{array}{l}\text { Parto } 1 \\
\text { Parto } 2 \\
\text { Parto } 3 \\
\text { Parto } 4\end{array}$} & 19 & 34.74 & \multirow{4}{*}{0.453} & \multirow{4}{*}{3} & \multirow{4}{*}{0.929} \\
\hline & & 23 & 34.93 & & & \\
\hline & & 17 & 33.26 & & & \\
\hline & & 10 & 38.60 & & & \\
\hline
\end{tabular}


Tabla 3. Prueba de Mann Whitney para comparación de concentraciones de lgG en calostro entre días posparto.

\begin{tabular}{|c|c|c|c|c|c|}
\hline $\begin{array}{c}\text { Días } \\
\text { posparto }\end{array}$ & $\mathbf{n}$ & $\begin{array}{c}\text { Rango } \\
\text { promedio }\end{array}$ & $\begin{array}{c}\text { Suma de } \\
\text { rangos }\end{array}$ & $\begin{array}{c}\text { U de Mann } \\
\text { Whitney }\end{array}$ & $\begin{array}{c}\text { Significancia } \\
\text { Bilateral }\end{array}$ \\
\hline $\begin{array}{l}\text { Día } 1 \\
\text { Día } 2\end{array}$ & $\begin{array}{l}32 \\
26\end{array}$ & $\begin{array}{l}33.91 \\
24.08\end{array}$ & $\begin{array}{c}1085.00 \\
626.00\end{array}$ & 275.000 & 0.027 \\
\hline $\begin{array}{l}\text { Día } 1 \\
\text { Día } 3\end{array}$ & $\begin{array}{l}32 \\
11\end{array}$ & $\begin{array}{l}23.66 \\
17.18\end{array}$ & $\begin{array}{l}757.00 \\
189.00\end{array}$ & 123.00 & 0.327 \\
\hline $\begin{array}{l}\text { Día } 2 \\
\text { Día } 3\end{array}$ & $\begin{array}{l}26 \\
11\end{array}$ & $\begin{array}{l}17.87 \\
21.68\end{array}$ & $\begin{array}{l}464.50 \\
238.50\end{array}$ & 113.500 & 0.140 \\
\hline
\end{tabular}

mismo con calcio, magnesio y azufre se encontró una correlación cercana a $(r=0,33 ; p<0,05)$. El incremento en la concentración de IgG en calostro, se acompaña de mayores concentraciones de minerales.

El espectro b del espectrofotómetro, colores azules y amarillos, presentó correlación positiva y significante con la concentración de IgG en calostro, pero no con las tonalidades rojo-verdes y la luminosidad. (Tabla 4)

La observación macroscópica del color relaciona los calostros de mejor calidad inmunológica a aquellos de color amarillo saturado.

La evaluación de la calidad del calostro mediante el color presentó correlación con b* y no con $\mathrm{L}^{*}(\mathrm{r}=-0,21$, $p=0,800)$, a diferencia de lo reportado por Gross et al., (2014) que presento correlación negativa significativa con $L^{*}(r=-0,45, p=0,0001)$.

\section{Conclusiones}

El calostro bovino de cruce Bos taurus $x$ Bos indicus con predominancia racial Gyr presentó calidades resaltables como la concentración de inmunoglobulinas, que permite clasificarlo de buena calidad. La población bovina evaluada tiene potencial para crear un banco de calostro del primer día posparto siendo el más apto para la transferencia de inmunidad pasiva.
La concentración de $\lg G$ en las muestras analizadas no se ve influenciada por el número de partos más sí por el número de días posparto. Es directamente proporcional a la concentración de proteína y densidad, base fundamental para el uso del calostrómetro en campo. En cuanto a los minerales presentó mayor correlación con la concentración de fósforo, fundamental dentro de las funciones metabólicas del desarrollo del animal.

Un calostro de buena o excelente calidad medido en el color es teóricamente un amarillo saturado, así como se observó en la evaluación de color el eje b*, eje de azules y amarillos, se comprobó su correlación con la concentración de IgG.

El avance en los procesos de investigación respecto al análisis de calidad de productos de origen animal, como el calostro, realizados en el piedemonte llanero forja conocimiento, progreso y evolución de los sistemas de producción bovina.

\section{Referencias}

Baumrucker CR, Burkett AM, Magliaro-Macrina AL, Dechow CD. Colostrogenesis: Mass transfer of immunoglobulin G1 into colostrum. J Dairy Sci. 2010;93:3031-3038.

Butler JE. Bovine Immunoglobulins: A Review. J. Dairy Sci. 1969; 52:1895-1909.

Tabla 4. Correlación de Spearman entre la concentración de IgG en calostro en los primeros tres días posparto y la luminosidad y colores a y $b$

\begin{tabular}{|l|c|c|c|}
\hline $\begin{array}{c}\text { Concentración de Ig G } \\
(\mathbf{m g} / \mathbf{m l})\end{array}$ & $\begin{array}{c}\text { Luminosidad } \\
(\mathbf{n m})\end{array}$ & $\begin{array}{c}\mathbf{a}^{*} \text { Rojo-verdes } \\
(\mathbf{n m})\end{array}$ & $\begin{array}{c}\mathbf{b}^{*} \text { Azules-amarillos } \\
(\mathbf{n m})\end{array}$ \\
\hline Coeficiente de correlación & $-0,021$ & 0,079 & $0,182^{*}$ \\
\hline Sig. (bilateral) & 0,800 & 0,332 & $\mathbf{0 , 0 2 4}$ \\
\hline
\end{tabular}


Chase CCL, Hurley DJ, Reber AJ. Neonatal Immune Development in the Calf and Its Impact on Vaccine Response. Vet Clin North Am Food Anim Pract. 2008;24:87-104.

Christiansen S, Guo M, Kjelden D. Chemical composition and nutrient profile of low molecular weight fraction of bovine colostrum. Int Dairy J. 2010;20:630-636.

Elfstrand L, Lindmark-Månsson H, Paulsson M, Nyberg L, Åkesson B. Immunoglobulins, growth factors and growth hormone in bovine colostrum and the effects of processing. Int Dairy J. 2002;12:879-887.

Flórez H, Martínez GP, Silva J, Romero A, Díaz E, Ruíz R, Donado P. 2000. Prevención de enfermedades y de la muerte de terneros en sistemas de doble propósito del trópico bajo colombiano. Corpoica Turipana. Colombia. Disponible en Internet: www. corpoica.org.co.

Foley JA, Otterby DE. Availability, Storage, Treatment, Composition, and Feeding Value of Surplus Colostrum: A Review1,2. J Dairy Sci. 1978;61:1033-1060.

Gross JJ, Kessler EC, Bruckmaier RM. Colour measurement of colostrum for estimation of colostral $\lg G$ and colostrum composition in dairy cows. J Dairy Res. 2014;81:440-444

Gulliksen S, Lie K, Sølverød L, Østerås O. Risk factors associated with colostrum quality in Norwegian dairy cows. J Dairy Sci. 2008; $91: 704-712$.

Hagiwara K, Kataoka S, Yamanaka H, Kirisawa R, Iwai H. Detection of cytokines in bovine colostrum. Vet Immunol Immunopathol. 2000;76:183-190.

Hernández-Castellano LE, Torres A, Alavoine A, Ruiz-Díaz MD, Argüello A, Capote J, Castro N. Effect of milking frequency on milk immunoglobulin concentration (IgG, $\lg M$ and $\lg \mathrm{A}$ ) and chitotriosidase activity in Majorera goats. Small Rumin. Res. 2011;98:70-72.

Hernández TE, Olaya JE. 2004. Caracterización inmunológica del calostro en dos predios del sistema doble propósito del Piedemonte Llanero y evaluación de cuatro métodos de conservación. trabajo de grado. Facultad de Ciencias Agropecuarias y Recursos Naturales. Escuela de Medicina Veterinaria y Zootecnia. Universidad de Los Llanos. Villavicencio. Meta. Colombia.

Kehoe S, Heinrichs A, Moody M, Jones C, Long M. Comparison of immunoglobulin $\mathrm{G}$ concentrations in primiparous and mul- tiparous bovine colostrum. The Professional Animal Scientist. 2011;27(3):176-180.

Liu GL, Wang JQ, Bu DP, Cheng JB, Zhang CG, Wei HY, Zhou LY, Zhou ZF, Hu H, Dong XL. Factors affecting the transfer of immunoglobulin G1 into the milk of Holstein cows. The Veterinary Journal. 2009;182:79-85.

Mee JF. Newborn Dairy Calf Management. Veterinary Clinics of North America: Food Animal Practice. 2008;24:1-17.

Muller LD, Ellinger DK. Colostral immunoglobulin concentrations among breeds of dairy cattle. J Dairy Sci. 1981;64:1727-30.

Oyeniyi O, Hunter A. Colostral constituents including immunoglobulins in the first three milkings postpartum. J Dairy Sci. 1978; 61:44-48

Pandey NN, Dar AA, Mondal DB, Nagaraja L. Bovine colostrum: A veterinary nutraceutical review. Journal of Veterinary Medicine and Animal Health, 2011;3(3):31-35.

Shoshani E, Rozen S, Doekes J. Effect of a short dry period on milk yield and content, colostrum quality, fertility, and metabolic status of Holstein cows. J Dairy Sci. 2014;97:2909-2922.

Tyler J, Steevens B, Hostetler D, Holle J, Denbigh JR J. Colostral immunoglobulin concentrations in Holstein and Guernsey cows. Am J Vet Res. 1999a; 60:1136-1139.

Tyler JW, Hancock DD, Thorne JG, Gay CC, Gay JM. Partitioning the mortality risk associated with inadequate passive transfer of colostral immunoglobulins in dairy calves. J Vet Intern Med. 1999b;13:335-337.

Vann R, Holloway J, Carstens G, Boyd M, Randel R. Influence of calf genotype on colostral immunoglobulins in Bos taurus and Bos indicus cows and serum immunoglobulins in their calves. J Anim Sci. 1995. 73:3044-3050.

Weaver DM, Tyler JW, Vanmetre DC, Hostetler DE, Barrington GM. Passive Transfer of Colostral Immunoglobulins in Calves. Journal of Veterinary Internal Medicine, 2000;14:569-577.

Epidat: programa para análisis epidemiológico de datos. Versión 4.1 Octubre de 2014. Consellería de Sanidade, Xunta de Galicia, España; Organización Panamericana de la Salud (OPS-OMS); Universidad CES, Colombia. Disponible en http://dxsp.sergas.es 